eISSN 2444-7986

DOI: http://dx.doi.org/10.14201/orl201673.14190

Artículo de revisión

\title{
ALTMETRICS PUEDEN NO SEGUIR SIENDO ALTERNATIVA POR MUCHO TIEMPO
}

\author{
Altmetrics can not remain a alternative for a long time \\ Julio ALONSO-ARÉVALO \\ Universidad de Salamanca. Biblioteca de Traducción y Documentación. Salamanca. España. \\ Correspondencia: alar@usal.es
}

Fecha de recepción: 29 de marzo de 2016

Fecha de aceptación: 13 de junio de 2016

Fecha de Publicación: 1 de julio de 2016

Conflicto de intereses: Los autores declaran no tener conflictos de intereses

Imágenes: Los autores declaran haber obtenido las imágenes con el permiso de los pacientes

Política de derechos y autoarchivo: se permite el autoarchivo de la versión post-print (SHERPA/RoMEO)

Licencia CC BY-NC-ND. Licencia Creative Commons Atribución-NoComercial-SinDerivar 4.0 Internacional

(c) Universidad de Salamanca. Su comercialización está sujeta al permiso del editor

RESUMEN

\begin{abstract}
Introducción y objetivo: La irrupción de internet y de los medios sociales está teniendo una poderosa influencia en las formas en que los investigadores descubren, acceden, procesan y comunican la información. Los métodos utilizados por los sistemas de medición del impacto de un documento científico utilizados durante los últimos cuarenta años, ya en entredicho, se están manifestando más claramente insuficientes en este nuevo contexto, ya que no proporcionan información de todas las interacciones que se producen en los nuevos medios sociales. Altmetrics surge como un recurso complementario y no excluyente de las métricas tradicionales para proporcionar una visión más rica de la influencia de una investigación. Método: Revisión narrativa. En este artículo se hace un análisis de las implicaciones de almetrics, ventajas, inconvenientes, y logros. Resultados: Se considera que altmetrics proporciona una visión más amplia de las implicaciones sociales de la investigación más allá de las puramente académicas. Discusión: Aún no es fácil desentrañar cual es el significado de altmetrics y qué tipo de impacto mide exactamente. Conclusiones: Altmetrics aunque aún no es considerado por las agencias de acreditación científica, ya está siendo utilizado por algunos de los más importantes editores, ya que proporciona una imagen inmediata del impacto de una investigación, y a la larga puede ser un mecanismos para predecir el futuro impacto académico de una investigación, aún quedan muchos aspectos a tener en cuenta como todos los relativos a la normalización de fuentes y métodos de compilación de los resultados, pero podemos decir que altmetrics ha llegado y tendrá que ser tenido en cuenta en un futuro inmediato por parte de los organismos de acreditación y financiación de la investigación.
\end{abstract}

PALABRAS CLAVE altmetrics; investigación; evaluación; impacto; redes sociales; investigación 2.0

SUMMARY Introduction and objective: The emergence of the Internet and social media is having a powerful influence on the ways in which researchers discover, access, process and communicate information. The methods used by systems measuring the impact of a scientific paper used during the last forty years, and questioned, are demonstrating more clearly insufficient in this new context, they do not provide information of all interactions that occur in the new social media. Altmetrics emerges as a complementary resource and does not exclude traditional metrics to provide a richer view of the influence of an investigation. Method: Narrative review. 
This article presents an analysis of the implications of altmetrics, advantages, disadvantages, and achievements made. Results: It is considered that altmetrics provides a broader view of the social implications of research beyond the purely academic. Discussion: While it is not easy to disentangle what is the meaning of altmetrics and what kind of impact accurately measures. Conclusions: altmetrics although it is not yet considered by the agencies of scientific accreditation, is already being used by some of the most important publishers, as it provides an immediate picture of the impact of an investigation, and ultimately may be a mechanism to predict the future academic impact of research, there are still many aspects to consider as all related to standardization of sources and methods of compilation of the results, but we can say that has altmetrics come and will have to be taken into account in the immediate future by Iso accreditation bodies and research funding.

KEYWORDS

altmetrics; research; evaluation; impact; social networking; research 2.0

Las agencias de evaluación y entidades financieras necesitan una medida objetiva de la calidad de las publicaciones, consecuentemente existe una demanda de medidas de este tipo. El factor de impacto (FI) —también conocido como índice de impacto-, es una medida de la importancia de una publicación científica. Cada año es calculada por el ISI Institute for Scientific Information- para aquellas publicaciones a las que da seguimiento, las cuales son publicadas en un informe de citas llamado Journal Citation Report (JCR). El FI tiene una influencia enorme, pero controvertida. Al ya tradicional Journal Citation Report del ISI se han ido sumando algunas otras alternativas bien en la forma de medir dicho impacto (índice de Hirsch, Scimago Journal Rank), bien en la cobertura temática o la de procedencia geográfica de las revistas (IN-RECS, IN-RECJ, RESH, IHCD...).

EI FI de una revista es la media de veces que, en un año determinado, fueron citados los artículos publicados por esta revista en los dos años anteriores.

El FI tiene algunas ventajas que hay que considerar: su cobertura internacional amplia con más de 8400 publicaciones de 60 países, los resultados son publicados y disponibles (FECYT) y es fácil de usar y entender.

Por ejemplo, el artículo de Lowry et al. [1], está considerado el artículo más citado de toda la historia de la ciencia (según el ISI Web of Science -WOS-, 1945-2010). En julio de 2005 ya contaba con 293.328 citas. Sin embargo Albert Einstein ha sido citado a día de hoy sólo 6647 veces. La revista con mayor índice de impacto de todas es Cancer Journal for Clinicians $(94,262)$. Este año Es- paña tiene 76 revistas con impacto (en 2009 tuvo 59 y en 2008 solo 37 ).

La Web of Science y su correspondiente factor de impacto son insuficientes para la comprensión del impacto de los trabajos académicos de aquellos países menos representados en el índice [2] pero, en contraposición, las métricas alternativas ofrecen una gran oportunidad para reorientar las estructuras de incentivos hacia los problemas que contribuyen al desarrollo o, al menos, a las prioridades locales. Se ha escrito mucho sobre las limitaciones metodológicas de los sistemas de medición del impacto de las publicaciones científicas y son los académicos de las regiones menos favorecidas los que están sufriendo las más graves consecuencias. Problemas derivados de los sesgos disciplinares, ya que, en estos índices, están más representadas las ciencias que las humanidades y las ciencias sociales, además el comportamiento de los usos de cita y obsolescencia son muy distintos en unas y otras áreas. Quizás los 2 años de medición que evalúa Web of Knowledge sean suficientes para las ciencias por su alto índice de obsolescencia, pero no para el resto de áreas de conocimiento. Sesgos lingüísticos, ya que fundamentalmente están representadas las lenguas anglosajonas respecto al resto; otras cuestiones importantes son las relativas a las áreas geográficas representadas, que tienen que ver con los países más desarrollados, e incluso con los emergentes, ya que la política de aceptación de revistas en Web of Knowdlge, si bien aparece establecida en su página y da unas pautas aparentemente claras, en la realidad no es tan transparente. Cada año Thomson Reuters evalúa aproximadamente 2.000 revistas para la inclusión de las mismas en Web of Science (en este enlace podrá encontrar información sobre los criterios de 
selección que utiliza Thomson Reuters para la inclusión de revistas ${ }^{1}$.

Una muestra de todo esto es un estudio de Kahn [3] sobre los documentos muy citados en Journal Citation Report. Estudia aquellos que se citaron al menos 1000 veces desde su publicación hasta 2011. Un total de 1857 fueron identificados en la base de datos Science Citation Index Expanded ${ }^{\mathrm{TM}}$ (Thomson Reuters, Nueva York) entre 1899 y 2011. Si se analiza la autoría, entre los autores más citados, en el $33 \%$ de los casos eran obras de un solo autor, el $61 \%$ eran de una sola institución y el $83 \%$ eran de un único país. Estados Unidos ocupa el primer lugar en los 6 indicadores. Los países de «G7» (Estados Unidos, Reino Unido, Alemania, Canadá, Francia, Japón e Italia) fueron escenario de casi todos los documentos muy citados. Las 12 instituciones más productivas son de Estados Unidos con la Universidad de Harvard y Texas como líderes. Las tres revistas más productivas fueron Chemical Reviews, Nature y $A n$ nual Review of Biochemistry.

En esta línea se han producido algunas declaraciones por parte de grupos numerosos de científicos. Es el caso de la «Declaración de San Francisco sobre Evaluación de la Investigación (DORA)», impulsada por la «Sociedad Americana de Biología Celular (BCSV)» junto con un grupo de directores y editores de revistas científicas, reconoce la necesidad de mejorar la forma en que se evalúan los resultados de la investigación científica [4]. El grupo se reunió en diciembre de 2012 durante la reunión anual de la BCSV en San Francisco e hizo circular un proyecto de declaración entre los diversos grupos de interés. Se trata de una iniciativa mundial que abarca todas las disciplinas académicas. Animamos a las personas y organizaciones que están preocupados por la evaluación adecuada de la investigación científica a que firmen DORA [3]. La declaración pone de manifiesto que existe una necesidad apremiante de mejorar la forma en que los resultados de la investigación científica son evaluados por las agencias de financiación, instituciones académicas y otros. Para solucionar este problema desarrolló una serie de recomendaciones invitando a los interesados

${ }^{1}$ http://wokinfo.com/publisher_relations/journals (c) Ediciones Universidad de Salamanca / CC BY-NC-ND [171] en todas las disciplinas científicas a que indiquen su apoyo al añadir sus nombres a la presente declaración.

Estas recomendaciones están dirigidas a los organismos de financiación, las instituciones académicas, las revistas, las organizaciones que suministran métricas y a los investigadores individuales:

- La necesidad de eliminar el uso de métricas basadas en revistas, tales como índice de impacto de revistas, en la financiación, en los nombramientos y en las consideraciones de promoción, la necesidad de evaluar la investigación por sus propios méritos y no en base a la revista en la que se publica la investigación y

- la necesidad de aprovechar las oportunidades que ofrece la publicación en línea (como relajar los límites innecesarios en el número de palabras, figuras y referencias en artículos, y la exploración de nuevos indicadores de la importancia y el impacto).

En este mismo sentido se ha pronunciado el International Council of Science que dice literalmente: «Quienes participan en la administración de la investigación utilizan métricas tradicionales para la evaluación de la importancia y el impacto de la investigación. Estas métricas a su vez afectan el comportamiento de investigadores, tales como la elección de revistas, ya que se busca maximizar su desempeño, como por las métricas utilizadas, lo que contribuye al mantenimiento de los altos precios de las publicaciones. La apertura y el compartir, permite un nuevo reconocimiento del impacto de la investigación a través de nuevas contribuciones y la generación de conjuntos de datos, software, código, blogs, wikis y foros. Las métricas utilizadas en la evaluación de la investigación y los investigadores debieran ayudar a promover el acceso abierto y la ciencia abierta, y la comunidad científica debe participar en el plenamente en su diseño» [5].

Por otra parte, los medios sociales están generando una influencia en los procesos de comunicación científica y en los hábitos y comportamientos de los investigadores de todas las disciplinas. La influencia generada por los medios sociales ha sido calificada como «Investigación 2.0», «Social Referen- 
ce» o «Altmetrics». Almetrics recoge las menciones de artículos académicos de todas partes de la web mediante la recopilación de menciones en los periódicos, blogs, redes sociales y otros sitios web. El aumento del interés en la medición del impacto social de la investigación ha llevado a la bibliometría clásica a lo que se ha denominado altmetrics o métricas alternativas. En este concepto se presta especial atención a una medición de impacto amplia, cuyo objetivo es descubrir el impacto que un conjunto de publicaciones tiene sobre grupos específicos de usuarios [6]. Si bien es necesario decir que altmetrics no es una medición excluyente respecto a los sistemas de medición bibliométrica, ya que más bien viene a complementar y no a sustituir la metodología de evaluación del impacto que se ha venido desarrollando durante el último medio siglo, altmetrics nos da una visión más amplia de ese impacto, el impacto social de la investigación, en qué medida esa investigación ha tenido una amplia repercusión social en los nuevos medios como blog, prensa, redes sociales.

Altmetrics presenta algunas ventajas adicionales y complementarias a la bibliometría ortodoxa. Generalmente los índices de citas como Web of Knowledge fundamentalmente se ocupan del impacto de los artículos de revistas, sin tener en cuenta la medición para otros productos como libros, presentaciones, capítulos, páginas web, etc. Muchas de las herramientas altmétricas ya se ocupan de estos otros formatos que, en algunas áreas de conocimiento como las humanidades y las ciencias sociales, son los principales vehículos de comunicación científica.

Por otra parte, como antes mencionamos, en la evaluación científica clásica hay una dilación de tiempo amplia entre el momento en que se publica el artículo y cuando este recibe las primeras citas que dependiendo de la disciplina pueden ser de varios meses a varios años. En el caso de las mediciones alternativas el documento recoge evidencias desde el mismo momento de la publicación. Es decir, desde el minuto cero ese artículo tendrá datos asociados en tiempo real de las veces que se ha visualizado, descargado mencionado en blog, redes sociales o prensa, o compartido en un gestor de referencias. Esta cuestión es importante en cuanto a la capacidad de poder predecir el futuro impacto de esa investigación. A este respecto se han realizado algunas investigaciones como las llevadas a cabo por Brody y Kurtz (2006) [7] que correlacionan las descargas de un documento en un repositorio en acceso abierto con las citas que recibe el documento, concluyendo que existe una clara correlación entre descargas y las citas recibidas con variaciones en función de la disciplina. Otra de estas investigaciones desarrolladas al respecto estudiaba en qué medida los tweets pueden predecir las futuras citas que va a recibir un documento [8]. La conclusión del estudio es que los tweets recibidos pueden predecir aquellos artículos que son más altamente citados dentro de los 3 primeros días de la publicación del artículo y que la actividad de los medios sociales o bien aumenta las citas o refleja las cualidades subyacentes del artículo para predecir futuras citas.

Otra de las críticas que se hacen a los índices de citas tradicionales es que la capacidad de citación entre disciplinas varía considerablemente y además influyen en ella diferentes factores externos como la ubicación geográfica o lingüística del investigador. Cuando buscamos citas o el impacto en herramientas, como Web of Knowledge de Thomson Reuters, no tiene en cuenta todas las revistas, sólo las incluidas en el índice, existiendo una ventaja competitiva para aquellas disciplinas más abiertamente científicas como las ciencias puras, biomedicina, etc.

También se dice que las mediciones altmétricas están menos sujetas a la manipulación por parte de autores y editores que las citas bibliográficas. Todos somos conscientes de las citas hechas a través de los llamados colegios invisibles con la finalidad de aumentar el número de citas, pero sin finalidad científica, igualmente ocurre con los editores, que en ocasiones exigen citar artículos de la propia revista para acrecentar el impacto. Aunque a decir verdad estos nuevos sistemas también pueden ser manipulados en alguna manera. Una investigación llevada a cabo por miembros del «Grupo EC3» de la Universidad de Granada fue capaz de crear un perfil falso de un autor también falso que emulaba a dos excelentes ciclistas Marco Pantani y Alberto Contador. El supuesto autor Marco Alberto Pantani-Contador, llegó a recibir más de medio centenar de falsas citas en el perfil de Google Schoolar Citations [9-11]. 
Otra de las ventajas del altmetrics se relaciona con su propia naturaleza, es decir que muestra la atención recibida por una obra más allá de las citas, es decir opera más allá del ámbito estrictamente académico para adentrarse en el impacto social de la investigación. Este es un concepto que aparece en casi todos los grandes sistemas de evaluación como EFQM o ISO. En este mismo sentido NISO [13] considera que las ventajas de altmetrics sobre el $\mathrm{FI}$ son que es abierto, rápido, se basa en fuentes múltiples y es fácil de usar y comprender.

La bibliometría tradicional no tiene en cuenta otros impactos, por ejemplo cómo ha sido utilizada esa publicación por parte del público, por instituciones, empresas, alumnos... Es decir no tiene en cuenta el resto del ecosistema académico. Por ello algunas agencias como Wellcome Trust o Research Excellence Framework (REF) animan a los investigadores a aumentar su compromiso con el uso de otros indicadores alternativos que reflejen una más amplia influencia.

Pero los nuevos sistemas de medición social presentan también algunas desventajas e incoherencias. Se dice que un alto número de menciones no es significativo de si la investigación es buena o mala, ya que se trataría de un índice de popularidad o de influencia social, pero no la calidad de una investigación. También existe una falta de consistencia y normalización entre los datos y sitios utilizados para la medición. Unas herramientas utilizan unas fuentes para la toma de datos y otras utilizan fuentes diferentes. Por ejemplo "Almetric.com» utiliza CiteUlike y otras herramientas como Plum o Impact Story no tienen en cuenta esta fuente. Casi todas ellas utilizan Mendeley, pero incluso en estos casos las mediciones que hacen una y otra plataforma difieren considerablemente, especialmente en el caso de tweets recibidos, menciones en Facebook, etc. Es decir existe una falta de normalización y una cierta incoherencia en las metodologías. A este respecto NISO publicó una primera aproximación a la normalización de datos "Alternative Assessment Metrics (Altmetrics) Initiative» ("Alternative Metrics Initiative - National Information Standards Organization» 2016) con el objetivo de establecer las bases para una normalización del impacto social de la investigación. Otra iniciativa de normalización en el campo de la biomedicina es la desarrollada por Becker Library Research Model for Assesment Research Impact, un intento de poner de acuerdo a los sitios web, editores e investigadores para establecer un modelo riguroso de aplicación de estos indicadores para que sean adoptados por agencias, comités e investigadores de manera que enriquezcan el discurso académico. Otra de las críticas viene dada por el hecho de que algunas de estas herramientas de la web social son muy volátiles como el caso del gestor de referencias Connotea que desapareció hace un par de años, y era considerado como fuente por plataformas como «Altmetric.com».

Si bien con internet y los avances en el análisis de big data, la asignación de identificadores persistentes como «ORCID», un identificador internacional de identificación de autores con rango de norma y que es capaz de interoperar con todos los sistemas desarrollados hasta la fecha para la identificación sin ambigüedad de los autores o «DOI», un identificador internacional de objetos digitales que funciona como una URL persistente, los sistemas científicos cada vez están más interconectados, lo que facilita que cada vez se avance más en hacer mediciones más exactas del impacto de la investigación. Las estadísticas de Mendeley, por ejemplo, parece proporcionar más conocimientos sobre el uso académico de un documento que Twitter.

También se dice que el impacto social puede variar mucho respecto al impacto académico de una publicación. Una investigación de Costas, et al. [12] hizo un análisis de la presencia de diferentes indicadores de métricas alternativas proporcionados por «Altmetric.com» en distintos campos científicos, centrándose especialmente en su relación con las citas. Los resultados confirman que la presencia y densidad de los recuentos altmétricos siguen siendo muy bajas y es muy frecuente entre las publicaciones científicas encontrar sólo entre un $15 \%$ a un $24 \%$ de publicaciones que presentan alguna actividad altmétrica concentrada en las publicaciones más recientes, aunque su presencia ha aumentado con el tiempo. Las publicaciones de las ciencias sociales, humanidades y las ciencias médicas y de la vida muestran la más alta presencia de indicadores altmétricos, indicando su valor potencial y el interés para estos campos. El análisis de las relacio- 
nes entre altmetrics y citas confirma afirmaciones anteriores de correlaciones positivas pero relativamente débiles, lo que apoya la idea de que altmetrics no refleja el mismo tipo de impacto que las citas. Además, los recuentos altmetrics no siempre presentan un mejor filtrado de las publicaciones más citadas de la revista. El valor de altmetrics como una herramienta complementaria de análisis de citas es evidente, aunque se sugiere que se debe investigar más profundamente sobre esta cuestión para desentrañar el significado y el valor de los indicadores para la evaluación de su potencial.

Durante los últimos años algunas plataformas y editores como Plos o Elsevier han adaptado a sus bases de datos a las medidas altmétricas. Esto también tiene que ver mostrarse innovadoras y con la adopción del modelo de acceso abierto denominado «el autor paga» con la finalidad de ofrecer un valor añadido a este modelo de negocio.

Algunas de las principales plataformas más importantes que monitorean la web y extraen datos de uso e influencia social de la investigación son:

ALMETRIC.COM. La plataforma de Scholastics, proporciona páginas de detalles de la atención recibida de una investigación en los medios sociales, a través de la instalación de un bookmarklet que permite la visualización de la "rosquilla» de colores de "Almetric.com» con los datos sobre la influencia de la investigación en diversos medios como Twitter, Facebook, Mendeley, blogs, prensa, etc. Hoy en día la base de datos "Altmetric.com» contiene menciones de más de 4 millones de fuentes de investigación (incluyendo artículos de revistas, bases de datos, imágenes, documentos, informes y más) y está en constante crecimiento. Para realizar un seguimiento de las menciones en línea para una parte específica de la investigación, se tiene en cuenta tres cuestiones:

- Un documento fuente (artículo de revista, informes, imágenes).

- Un identificador (DOI, RePeC, PMID, Arxiv ID, SSRN...).

- Menciones recibidas. Estos identificadores también ayudan a reconocer las diferentes versiones de una investigación y a su desambiguación. Por ejem- plo, un artículo de una revista puede estar originalmente disponible en una plataforma editorial y con un DOI, y luego estar alojado en PubMed o en un repositorio institucional. La puntuación de «Altmetric.com» es calculada automáticamente, por conteo ponderado en función de la atención recibida por la investigación basada en 3 factores principales:

1. Volumen. Número de menciones.

2. Fuente. Cada categoría es puntuada de diferente forma. Por ejemplo puntúa más la mención en un artículo de prensa que en un blog.

3. Autores. Puntúa en función de la categoría del autor que haya hecho la medición, o en que revista se haya hecho en función de su impacto o audiencia.

En conjunto, el resultado final de la puntuación representa una aproximación ponderada de todas las menciones que se han hecho en diferentes medios a esa investigación. El algoritmo «Altmetric.com» calcula una puntuación global teniendo en cuenta el volumen, la fuente y el autor en función de las menciones que recibe un documento. Esto incluye las menciones de artículos académicos sobre los sitios de medios sociales (por ejemplo Twitter, Facebook, Pinterest, Google+), blogs científicos, muchos medios de comunicación convencionales, incluyendo The New York Times, The Guardian, las publicaciones en idiomas distintos al inglés, como Die Zeit y Le Monde y publicaciones de interés especial como Scientific American y New Scientist.

Plum Analytics. Plum, que significa cereza, es el portal de métricas de investigación de Ebsco. Realiza un seguimiento de todos los productos de la investigación en cualquier forma, proporcionando una poderosa herramienta que aumenta la capacidad de la métrica tradicional. La herramienta PlumX reúne a las métricas a través de varias categorías: citaciones, uso, menciones y capturas recogidas de los datos proporcionados por los medios sociales. Los documentos incluyen: artículos, entradas del blog, capítulos de libros, libros, casos, ensayos clínicos, comunicaciones a congresos, conjuntos de datos, cifras, subvenciones, entrevistas, cartas, medios de comunicación, patentes, posters, presenta- 
ciones, código fuente, tesis, disertaciones, vídeos, páginas web. Plum proporciona datos objetivos sin establecer ponderaciones o ranking como «Altmetric.com». También es posible añadir widgets de PlumX a su repositorio institucional, perfiles de investigadores, sitios web del departamento o blogs, etc.

IMPACT STORY. A cambio de una cuota mensual se pueden tener perfiles de ImpactStory, con un período de prueba gratuita inicial, que permite al investigador construir un currículum vitae en línea que proporciona datos sobre la atención recibida de sus trabajos en los medios sociales.

PLOS METRICS. Las métricas PLOS están libremente disponibles directamente desde las páginas de artículos publicados en PLOS.

En conclusión altmetrics aporta una serie de beneficios para los investigadores [14]. Con altmetrics los autores podrán disponer, además de las citas y el impacto de sus publicaciones, del impacto social de las mismas en otros contextos. Facilitando gestionar el control de su identidad digital y su reputación en línea. Una investigación no sólo tiene influencia en su resultado final, a veces existen otros documentos que la acompañan como presentaciones o set de datos, de los que no se recogen evidencias en las mediciones tradicionales. En segundo lugar disponer de evidencias más allá de las facilitadas por las métricas tradicionales en un mundo tan competitivo como el actual, donde los presupuestos de financiación no se incrementan y sí los concurrentes a una acreditación o financiación, facilitará al investigador alcanzar su propósito. Poder demostrar un amplio impacto ayudará a hacerse una idea más completa del potencial de esa investigación. Además estás medidas de tracción ayudarán a tener un mayor impacto debido a los mecanismos de visualización que incidirán en su retroalimentación. $Y$ en tercer lugar altmetrics es una herramienta orientada a descubrir la investigación. Ya que ayudará a visualizar aquellas propuestas que han recibido una mayor atención por parte de los medios sociales. Y también ayudará a los investigadores a identificar los mejores canales de influencia de sus publicaciones.

Algo se está moviendo en el contexto:

- Hoy en día incluso multinacionales de la información científica como Elsevier, que ha sido durante mucho tiempo un defensor a ultranza de la infometría robusta. está interesándose en la comprensión de cómo se pueden utilizar estas nuevas medidas en relación con los datos de uso y citación, para proporcionar nuevos indicadores significativos para la comunidad investigadora. Altmetrics ofrecen una visión alternativa sobre el uso e impacto de la investigación por parte de los lectores de artículos académicos y esta información ha impulsado a autores, investigadores y editores a tratar de entender cuál es la importancia de estos datos. Por ello, Elsevier ha empezado a participar en el proyecto NISO Altmetrics y esta línea sigue en su proyecto Article Usage Reports, un programa que informa a los autores que han publicado en las revistas de Science Direct y «Elsevier.com» sobre cómo se está visualizando su artículo.

También en la $14^{a}$ edición del «Ranking Web de Repositorios», (http://repositories.webometrics.info/es) en su última versión de 2014 incluye mediciones altmetrics de las siguientes fuentes en el indicador visibilidad: Academia, Facebook, Linkedln, Mendeley, ResearchGate, Slideshare, Twitter, Wikipedia (todas las ediciones), Wikipedia (versión Inglés) y YouTube (25\%).

Otro ejemplo es Plos con el modelo ALMs de PLoS Article Level Metrics (https://universoabierto.com/2015/12/31 larticle-level-metrics-almetrics-en-plos/) que se encarga de hacer la recopilación de estas evidencias tales como su cobertura en blogs, cobertura en noticas y presentarla a sus lectores.

- Taylor \& Francis también incluye datos almétricos en su Top 20.

MIT Press también está incorporando medidas altmétricas en sus publicaciones de Batches Books Series. Los departamentos de marketing y publicación utilizan estas medidas para poder tener datos fiables y determinar el éxito de sus páginas, conocer la procedencia geográfica de uso y de esta manera establecer estrategias de mercado.

- En instituciones como Michigan Publishing, altmetrics contribuye a disponer de información sobre las publicaciones en tiempo real para demostrar el valor 
de la actividad editorial y de esta manera justificar la inversión llevada a cabo por la entidad pública. Por ello también ha incorporado medidas altmétricas a su programa de publicación de libros en acceso abierto -Digital Culture Books - y al repositorio institucional Deep Blue. Esto está siendo especialmente útil para los libros que no disponían de $\mathrm{Fl}$ y de este modo tienen información sobre su uso. Teniendo de esta manera evidencias que puedan justificar la inversión llevada a cabo ante los organismos de financiación.

Todos estos signos indican que altmetrics pueden no seguir siendo alternativa por mucho tiempo, lo que es especialmente atractivo para aquellas disciplinas y áreas que tradicionalmente han sido excluidas del análisis bibliométrico, ya que no están suficientemente representadas en bases de datos como Web of Science (WoS) o Scopus, como las ciencias sociales y las humanidades.

La naturaleza del impacto de la investigación es cada vez más amplia y multifacética que nunca. La incorporación junto a la medición ortodoxa de medidas alternativas presenta una oportunidad para obtener una visión más holística del impacto de la investigación y su influencia, mediante la factorización de nuevos métodos de capturar e informar sobre la comunicación en línea y la actividad que rodea investigación. La combinación de altmetrics y bibliometría presenta una gran oportunidad para obtener una imagen más precisa del alcance y la influencia de los documentos de investigación en la era digital.

El valor de altmetrics como una herramienta complementaria de análisis de citas es evidente, aunque se sugiere que se debe investigar más profundamente sobre esta cuestión para desentrañar el significado y el valor de los indicadores para la evaluación de su potencial. La combinación de altmetrics y bibliometría presenta una gran oportunidad para obtener una imagen más precisa del alcance y la influencia de los documentos de investigación en la era digital.

Pero para que esto sea una realidad es muy importante entender las similitudes potenciales o diferencia en las cifras a través de diferentes plataformas y agregadores altmetrics.
Para ello es necesario recurrir a las mejores prácticas en la recopilación de datos altmétricos tanto por parte de los proveedores como de los agregadores y editores. Asimismo, resulta muy conveniente desarrollar normas, directrices y recomendaciones para introducir transparencia y coherencia a través de proveedores y agregadores.

\section{BIBLIOGRAFÍA}

1. Lowry $\mathrm{OH}$, Rosenbrough NJ, Farr $\mathrm{AL}$, Randall RJ. Protein Measurement with the Folin Phenol Reagent. The Journal of Biological Chemistry (JBC). 1951;193: 265-75.

2. Alperin JP. Altmetrics could enable scholarship from developing countries to receive due recognition. LSE - London School of Economics and Political Science. 2014. Disponible en: http://blogs.Ise.ac.uk/impactofsocialscie nces/2014/03/10/altmetrics-fordeveloping-regions/. [Citado el 13 de junio de 2016].

3. Ho YS, Kahn M. A bibliometric study of highly cited reviews in the Science Citation Index expanded ${ }^{\mathrm{TM}}$." Journal of the Association for Information Science and Technology. 2014;65(2):372-385. Disponible en: http://onlinelibrary.wiley.com/doi/10.100 2/asi.22974/abstract;jsessionid=E8FC0 D669AE94899786856E2B0A10AA5.f04 t04. [Citado el 13 de junio de 2016].

4. Declaration on Research Assessment (DORA). Disponible en: http://www.observatorioderevistascientif icas.com/index.php/noticias/234declaracion-de-san-francisco-sobreevaluacion-de-la-investigacion-dora. [Citado el 13 de junio de 2016].

5. International Council of Science. Open access to scientific data and literature and the assessmentof research by metrics [en línea]. S.I.: International Council of Science, 2014.. Disponible en: http://www.icsu.org/generalassembly/31st-general-assemblyauckland-2014/news/ICSU Report on Open Access.pdf. [Citado el 13 de junio de 2016]. 
6. Bornmann L. [e-Book] Which people use which scientific papers? An evaluation of data from F1000 and Mendeley, Max Planck Institute, 2015.

7. Kurtz M, Brody T. The impact loss to authors and research. Open Access: Key Strategic, Technical and Economic Aspects. 2006; (5). Disponible en: http://eprints.soton.ac.uk/40867/2/chapt er5distro.pdf. [Citado el 13 de junio de 2016].

8. Eysenbach G. Can tweets predict citations? Metrics of social impact based on Twitter and correlation with traditional metrics of scientific impact. J Med Internet Res. 2011;13(4):e123.

9. Delgado López-Cózar E, RobinsonGarcia N, Torres-Salinas D. Manipulating Google Scholar Citations and Google Scholar Metrics: simple, easy and tempting. 2012. arXiv:1212.0638 [cs]. Disponible en: http://arxiv.org/abs/1212.0638. [Citado el 13 de junio de 2016].

10. Delgado López-Cózar E, RobinsonGarcia N, Torres-Salinas D. The Google Scholar Experiment: how to index false papers and manipulate bibliometric indicators. Journal of the American Society for Information Science and Technology. 2013. Disponible en: http://arxiv.org/ftp/arxiv/papers/1309/13 09.2413.pdf. [Citado el 13 de junio de 2016].

11. Delgado López-Cózar E, RobinsonGarcia N, Torres-Salinas D. Science
Communication: Flawed Citation Indexing. Science. 2013;342(6163):1169. Disponible en: http://science.sciencemag.org/content/3 42/6163/1169.2. [Citado el 13 de junio de 2016].

12. Hodgson C. NISO Altmetrics Standards Project White Paper. Altmetrics Steering Group. 2014 Disponible en: http://www.niso.org/apps/group_public/ down-

load.php/13295/niso_altmetrics_white paper_draft_v4.pdf. [Citado el $\overline{13}$ de junio de 2016].

13. Costas R, Zahedi Z, Wouters P. Do "altmetrics" correlate with citations? Extensive comparison of altmetric indicators with citations from a multidisciplinary perspective. Journal of the Association for Information Science and Technology. 2014. Disponible en: http://doi.org/10.1002/asi.23309. [Citado el 13 de junio de 2016].

14. Williams C, Padula D. The Evolution of Impact Indicators: From bibliometrics to altmetrics. Almetric.com Scholastica. $2015 . \quad$ Disponible en: http://scholasticahq.com/altmetrics-theevolution-of-impact-indicators. [Citado el 13 de junio de 2016].

\section{ENLACES RELACIONADOS}

Universo Abierto:

http://www.universoabierto.com.

Universo Abierto:

https://universoabierto.com/2016/01/11/elsevi er-expande-sus-perspectivas-metricas-con-ellanzamiento-de-proyecto-piloto-altmetrics/. 